Article

\title{
The Relationship between Metabolically Healthy Obesity and the Risk of Cardiovascular Disease: A Systematic Review and Meta-Analysis
}

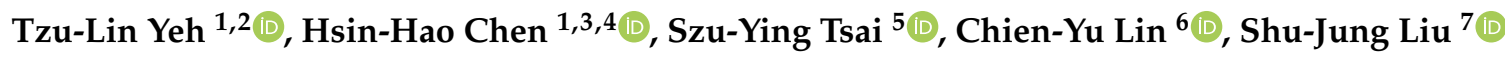 \\ and Kuo-Liong Chien ${ }^{2,8, *}$ \\ 1 Department of Family Medicine, Hsinchu MacKay Memorial Hospital, No. 690, Section 2, Guangfu Road, \\ East District, Hsinchu City 30071, Taiwan \\ 2 Institute of Epidemiology and Preventive Medicine, National Taiwan University, No.17, Xu-Zhou Rd., \\ Taipei City 10055, Taiwan \\ 3 MacKay Junior College of Medicine, Nursing, and Management, No. 92, Shengjing Rd., Beitou Dist., \\ Taipei City 11260, Taiwan \\ 4 Department of Medicine, MacKay Medical College, No.46, Sec. 3, Zhongzheng Rd., Sanzhi Dist., \\ New Taipei City 25245, Taiwan \\ 5 Department of Family Medicine, MacKay Memorial Hospital, No. 92, Section 2, Zhongshan North Road, \\ Taipei City 10449, Taiwan \\ 6 Department of Pediatrics, Hsinchu MacKay Memorial Hospital, No. 690, Section 2, Guangfu Road, \\ East District, Hsinchu City 30071, Taiwan \\ 7 Department of Medical Library, MacKay Memorial Hospital, Tamsui Branch, No. 45, Minsheng Road, \\ Tamsui District, New Taipei City 25160, Taiwan \\ 8 Department of Internal Medicine, National Taiwan University Hospital, No. 7, Zhongshan S. Rd., \\ Zhongzheng Dist., Taipei City 10002, Taiwan \\ * Correspondence: klchien@ntu.edu.tw; Tel.: +886-2-3366-8017
}

Received: 16 July 2019; Accepted: 12 August 2019; Published: 15 August 2019

\begin{abstract}
Cardiovascular disease (CVD) risk in individuals with metabolically healthy obesity (MHO) is unclear. We searched databases from inception to May 2019. Data were pooled using a random effects model. Newcastle-Ottawa Scale assessment was performed. Primary and secondary outcomes were CVD risk and all-cause mortality. Forty-three studies involving 4,822,205 cases were included. The median percentage of females, age and duration of follow-up was $52 \%, 49.9$ years and 10.6 years, respectively. The mean Newcastle-Ottawa Scale score of the articles was $7.9 \pm 1.0$. Compared to individuals with a metabolically healthy normal weight, individuals with MHO had higher adjusted risk of CVD and all-cause mortality. We identified a significant linear dose-response relationship between body mass index (BMI) and CVD risk among metabolically healthy individuals $(p<0.001)$; every unit increase in BMI increased the CVD risk. Multivariate meta-regression analysis showed that an increased proportion of women and age resulted in the risk of CVD affected by MHO reduction $(p=0.014, p=0.030$, respectively). Age and sex explained the observed heterogeneity and reported the adjusted $R^{2}$. MHO resulted in a significantly increased risk for CVD; therefore, long-term weight loss should be encouraged.
\end{abstract}

Keywords: metabolically healthy obesity; cardiovascular disease; all-cause mortality; meta-analysis

\section{Introduction}

According to the World Health Organization, cardiovascular disease (CVD) is the leading cause of mortality worldwide, with a rate of 17.7 million deaths per year, which continues to increase every year [1]. Based on the Global Burden of Disease Study, the overall global cardiovascular mortality rate 
increased by nearly $41 \%$ between 1990 and 2013 [2]. Obesity is an independent risk factor for CVD and all-cause mortality [3-6] due to the various physiological and metabolic changes that are associated with the condition. However, the risk for CVD differs between different obesity phenotypes $[7,8]$.

Obesity is not a uniform condition [8-10]. Generally, obesity is categorized into four phenotypes according to metabolic and anthropometric status: metabolically unhealthy obesity (MUO), metabolically unhealthy normal weight, metabolically healthy obesity (MHO), and metabolically healthy normal weight (MHNW) [9]. Individuals with MHO have been shown to have a lower risk of CVD and mortality compared with those with MUO [11,12]. However, it is unclear whether MHO negatively affects health [13] — an uncertainty that is compounded by the lack of consensus regarding the definition of MHO [14]. The concept of MHO was first introduced in 2001, when it was reported that some individuals with obesity do not have any outcomes of poor health [15]. Obesity is usually defined by body mass index (BMI), waist circumference (WC), or body fat. The term MHO is used to describe obesity in which insulin sensitivity [16], blood pressure, glucose level, and lipid profiles [17] are normal, and there is no diagnosis of metabolic syndrome based on the criteria of the National Cholesterol Education Program, Adult Treatment Panel III (ATP III) [18], International Diabetes Federation (IDF) [19], Joint Interim Statement (JIS) Harmonized Criteria of the IDF [20], or other criteria $[13,21]$. The prevalence of MHO varies from $2 \%$ to $28 \%$ and is affected by metabolic criteria as well as sex, age, smoking, region, and alcohol consumption [22].

Two recent meta-analyses reported that, compared with participants with MHNW, those with MHO were at higher risk of cardiovascular events but not all-cause mortality [23,24]. In 2016, MHO was introduced as a Medical Subject Heading term to describe a metabolically "benign" obesity that is associated with a "risk" of CVD [25]. A meta-analysis was performed in 2019, focusing on the comparison of the four phenotypes of obesity [26]; however, limited articles discussing MHO were found by a limited keyword search and the results were not significant. To the best of our knowledge, there have been very few studies addressing the relationships between these phenotypes and risks for CVD or other morbidities with comprehensive evidence.

If a more coherent definition of MHO could be agreed upon, it could enable clarification of whether this phenotype is beneficial or harmful to individuals [27] Thus, the present study aimed to perform a comprehensive systematic review and meta-analysis to evaluate the relationship between MHO and CVD.

\section{Methods}

We conducted a systematic review and meta-analysis following a pre-established protocol registered on PROSPERO (CRD 42019130244), reported in accordance with PRISMA guidelines [28]. (Table S1).

\subsection{Definition of Metabolic Health and Outcomes}

We extracted data relating to adults aged 18 years or older. Obesity was defined by BMI, WC, and body fat. Metabolic status was defined by insulin resistance, metabolic syndrome or metabolic disease diagnosed by blood glucose, blood pressure, or lipid profiles. We reported the outcomes for MHO compared with MHNW. The primary outcome was CVD as a composite of all fatal and nonfatal coronary heart disease (CHD), myocardial infarction (MI), stroke, heart failure (HF), and peripheral artery occlusion disease. The secondary outcome was all-cause mortality.

\subsection{Data Sources and Search Strategy}

In the present meta-analysis, we used comprehensive keywords to search large databases, adopted strict definitions, and utilized the PICO search tool. Two authors conducted the searches independently, and disagreements were resolved through discussion with the third author. Full search strategies are detailed in Table S2. Briefly, we conducted electronic searches of the following databases, supplemented with hand-searching, from inception to May 2019: PubMed/Medline, EMBASE, Cumulative Index to 
Nursing and Allied Health Literature, and the Cochrane database. We used the keyword (MHO) to identify articles published after 2016 and keywords (obesity OR body mass index) AND (metabolic) AND (normal OR healthy OR benign) to identify earlier articles. Outcomes were identified using the keywords (CVD OR CHD OR MI OR stroke OR HF) AND (morbidity OR morbidities OR mortality OR incidence). We did not place constraints on language, year of publication, or participant characteristics (including participant age) in order to ensure a comprehensive search and identify articles that are aligned with our results of PICO hand-searches. Letters and editorials were excluded. We contacted authors to obtain additional information if necessary.

\subsection{Study Selection and Methodological Quality Assessment}

Inclusion criteria were studies on adults with obesity and normal metabolic status, studies that reported the outcome measures of interest as primary or secondary outcomes of the paper, and cohort studies. Exclusion criteria were duplicate publications, irrelevant articles, studies where MHO and metabolically healthy overweight were not clearly defined, studies that did not provide a comparison with individuals with MHNW, and articles reporting case series, cross-sectional studies, or reviews. We did not include data relating to outcome measures other than those stated above, such as transition to MUO or incidence of diabetes, metabolic syndrome, or other heart disease such as atrial fibrillation, diastolic dysfunction, myocardial function, subclinical atherosclerosis, and subclinical myocardial ischemia.

The Newcastle-Ottawa Scale evaluates the quality of nonrandomized studies by the quality of selection, comparability, and outcome [29]. After initial screening, two authors independently scored the selected studies using this scale. If the two authors disagreed, agreement was reached by consensus with the third author. Details of the scoring system are provided in Table S1.

\subsection{Data Extraction}

Four authors independently extracted the following data: last name of the first author, year of publication, participants' characteristics, definition of obesity, definition of metabolic health, variables that were adjusted, definition of outcomes, and major findings (Table S2).

\subsection{Statistical Analyses}

For continuous outcomes, data were analyzed using the odds ratio (OR) with 95\% confidence intervals (CIs). Study-level information are presented as medians with ranges. All analyses were carried out using R software version 1.1.456 [30]. Assuming that the true effect size was not the same, we employed a random-effects model using DerSimonian and Laird's methods [31]. Results are presented in forest plots. Heterogeneity was quantified using the Cochran $Q$ test and $I^{2}$ statistics [32] and explained by prespecified subgroup analyses. For cumulative meta-analysis, the included studies were arranged in chronological order; then, multiple meta-analyses were performed by grouping studies by study year. We conducted a dose-response analysis to evaluate the linear relationships between BMI and the outcomes. We extracted data on BMI, number of participants, and person-years. The lowest boundary was assigned to the first BMI category (normal weight), as the reference group. The midpoint values of the BMI categories of overweight or obesity were used as the corresponding doses of outcomes. If the category had no upper boundary, the corresponding BMI was calculated as the lower boundary plus 1.5 times the range of the neighboring category. We estimated study-specific linear trends between BMI and the outcomes using a method developed by Greenland and Longnecker [33], then pooled the trends for random-effects meta-analysis. Weight-adjusted multivariate meta-regression models were used to test the contributions of effect modifiers (age, sex, follow-up duration, and smoking) [34]. Adjusted $R^{2}$ is commonly used to quantify the goodness of fit of our model in percentage $(0-100 \%)$. We assessed small-study effects using funnel plots and Egger's test [35]. Sensitivity analyses were conducted by considering the quality of the included studies, omitting each study and excluding $\mathrm{CV}$ mortality from $\mathrm{CV}$ morbidity in turn to test the robustness of the results. 


\section{Results}

\subsection{Description of Studies and Quality Assessment}

Figure 1 illustrates the search process. A total of 43 cohort studies were included; all included and excluded studies are listed in Table S3. The characteristics of the included studies are shown in Table S2. One article presented the hazard ratio of $\mathrm{MHO}$ and $\mathrm{MHNW}$ with metabolically healthy overweight as the reference; however, we could not obtain the correlation of MHO and MHNW after contacting the authors and thus excluded the study [36]. All of the included studies were published after 2004, and most were conducted in the United States or Europe. In total, 4,822,205 participants were included, with a median prevalence of $\mathrm{MHO}$ of $6.6 \%$ (range, $1.2-31.0 \%$ ). The median participant age was 49.9 (30.3-74.0) years; the median proportion of women was $52.0 \%(0-100 \%)$; and the median smoking rate was 20\% (5.7-67.6\%). The median follow-up duration was $10.6(1.0-30.0)$ years.

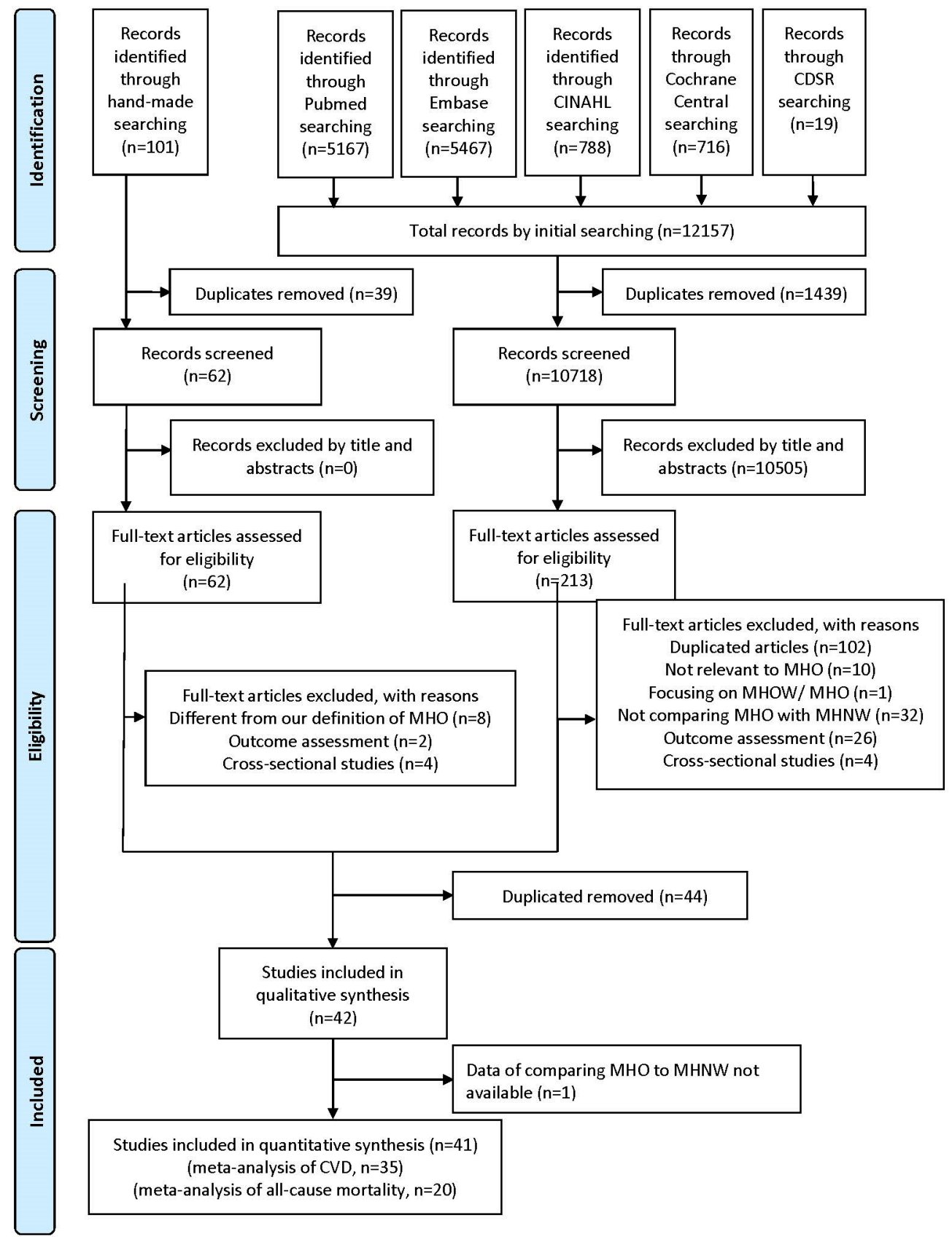

Figure 1. Flowchart of the study selection process. 
CDSR, Cochrane Database of Systematic Reviews; CINAHL, Cumulative Index to Nursing and Allied Health Literature; MHO, metabolically healthy obesity; MHOW, metabolically healthy overweight; MHNW, metabolically healthy normal weight.

The mean score ( \pm standard deviation) of the included studies according to the Newcastle-Ottawa Scale was $7.9 \pm 1.0$, out of a possible score of 9 (Tables S1 and S2). Most of the included studies had a quality score higher than 7. A study published in 2004 had the lowest score of 5, as the study did not adjust for smoking, assessed CVD outcome by telephone or mail contact, patients were followed up for 3.5 years only, and the dropout rate was not reported [37]. Two studies were scored 6; one of which used self-reported BMI and did not report baseline CVD or dropout rate [38] and the other determined CVD using an epidemiological questionnaire with follow-up of only 3.2 years, with no report of dropout rate [39]. We did not exclude any of the articles with quality scores below 7, but we performed sensitivity analysis to establish any effects of their inclusion.

\subsection{Results of the Meta-Analysis}

To evaluate the primary outcome of CVD, 35 cohort studies were pooled for the meta-analysis. Five of our included studies reported CV mortality as their CVD endpoint, which was part of our secondary outcome, all-cause mortality which refers to death of any reason. The cumulative forest plot showed an increase in the risk of CVD since 2005 (Figure 2). Participants with MHO were at significantly higher risk of CVD than individuals with MHNW (Table 1). Because of the underlying heterogeneity of definitions and outcomes, we performed subgroup analysis. Some of the studies used modified criteria of metabolic syndromes, which we aggregated for the purposes of the present study. Compared with participants with MHNW, those with MHO were at significantly higher risk of CVD, as defined by the modified ATP III criteria, modified IDF criteria, insulin resistance, modified JIS criteria, and other definitions. The forest plot is shown in Figure S1.

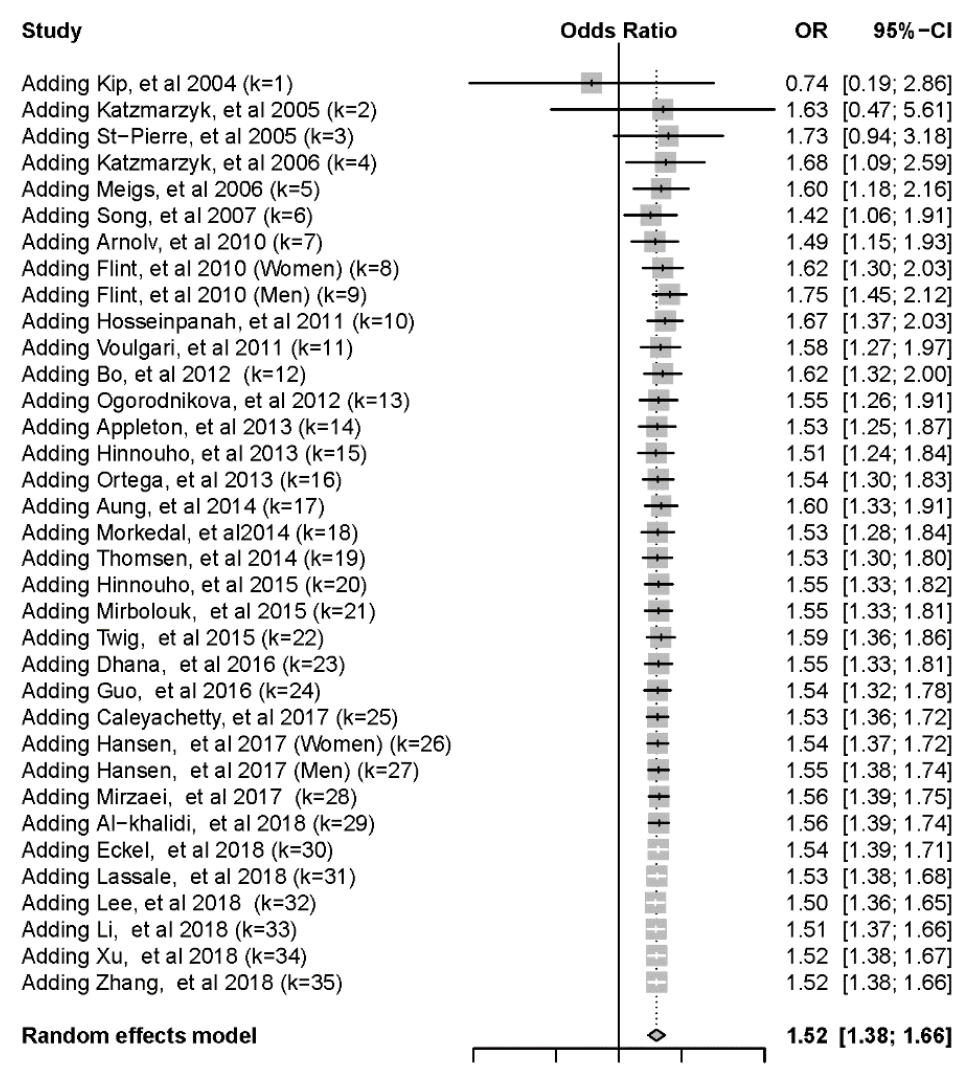

Figure 2. Cumulative forest plot of risk of cardiovascular disease from all included studies. CI, confidence interval; OR, odds ratio. 
Table 1. Risk of Cardiovascular Disease and All-cause Mortality Associated with Metabolically Healthy Obesity in Different Subgroup Analyses.

\begin{tabular}{|c|c|c|c|c|c|c|c|c|}
\hline & \multicolumn{4}{|c|}{ Risk of Cardiovascular Disease } & \multicolumn{4}{|c|}{ All-Cause Mortality } \\
\hline & OR $(95 \%$ CI $)$ & $\begin{array}{c}p \text { Value for } \\
\text { between-Group } \\
\text { Differences }\end{array}$ & $I^{2}(\%)$ & $\begin{array}{l}\text { Number of } \\
\text { Studies }\end{array}$ & OR $(95 \%$ CI) & $\begin{array}{c}p \text { Value for } \\
\text { between-Group } \\
\text { Differences }\end{array}$ & $I^{2}(\%)$ & $\begin{array}{l}\text { Number of } \\
\text { Studies }\end{array}$ \\
\hline Overall & $1.52(1.38 ; 1.66)$ & & 61 & 35 & $1.23(1.05 ; 1.43)$ & & 62 & 20 \\
\hline \multicolumn{9}{|l|}{ Subgroups } \\
\hline Definitions of metabolic health & & 0.17 & & & & 0.32 & & \\
\hline Modified ATP-III & $1.43(1.10 ; 1.85)$ & & 63 & 11 & $1.29(1.00 ; 1.66)$ & & 0 & 5 \\
\hline Modified IDF & $1.30(1.06 ; 1.60)$ & & 53 & 5 & NA & & NA & NA \\
\hline Insulin resistance & $1.72(1.30 ; 2.26)$ & & 0 & 5 & $1.56(1.19 ; 2.05)$ & & 12 & 5 \\
\hline Modified JIS & $1.45(1.23 ; 1.72)$ & & 19 & 5 & $1.12(0.78 ; 1.62)$ & & 77 & 4 \\
\hline Others & $1.79(1.49 ; 2.16)$ & & 77 & 9 & $1.10(0.81 ; 1.49)$ & & 76 & 6 \\
\hline Definitions of obesity & & 0.83 & & & & 0.27 & & \\
\hline Body mass index & $1.52(1.38 ; 1.67)$ & & 62 & 34 & $1.20(1.02 ; 1.43)$ & & 64 & 18 \\
\hline Waist circumference & $1.40(0.65 ; 2.98)$ & & NA & 1 & $1.45(1.09 ; 1.94)$ & & 0 & 2 \\
\hline Different outcomes & & 0.14 & & & & & & \\
\hline $\begin{array}{l}\text { Coronary heart } \\
\text { disease/myocardial infarction }\end{array}$ & $1.39(1.17 ; 1.65)$ & & 49 & 7 & & & & \\
\hline CVD mortality & $1.64(1.12 ; 2.39)$ & & 0 & 5 & & & & \\
\hline Fatal and nonfatal CVD & $1.57(1.40 ; 1.77)$ & & 68 & 22 & & & & \\
\hline Heart failure & $0.41(0.11 ; 1.48)$ & & NA & 1 & & & & \\
\hline
\end{tabular}

ATP III, National Cholesterol Education Program, Adult Treatment Panel III; CI, confidence interval; CVD, cardiovascular disease; IDF, International Diabetes Federation; JIS, joint interim statement; NA, not applicable; OR, odds ratio. 
Compared with participants with MHNW, those with MHO were at significantly higher risk of CVD when defined by BMI, but not when MHO was defined by WC (Table 1). The forest plot is shown in Figure S2. We used the composite outcome of CVD comprising all fatal and nonfatal CHD, MI, and HF. Compared with participants with MHNW, those with MHO were at significantly higher risk of CHD/MI, CVD mortality, and fatal and nonfatal CVD, but not HF (Table 1). The forest plot is shown in Figure S3.

A total of 11 articles with 35 BMI categories were pooled for dose-response analysis, which revealed a significant linear relationship between BMI and the risk of CVD $(p<0.001)$. For every unit increase in BMI, the risk of CVD increased by $2 \%$ (OR for slope, 1.019; Figure 3).

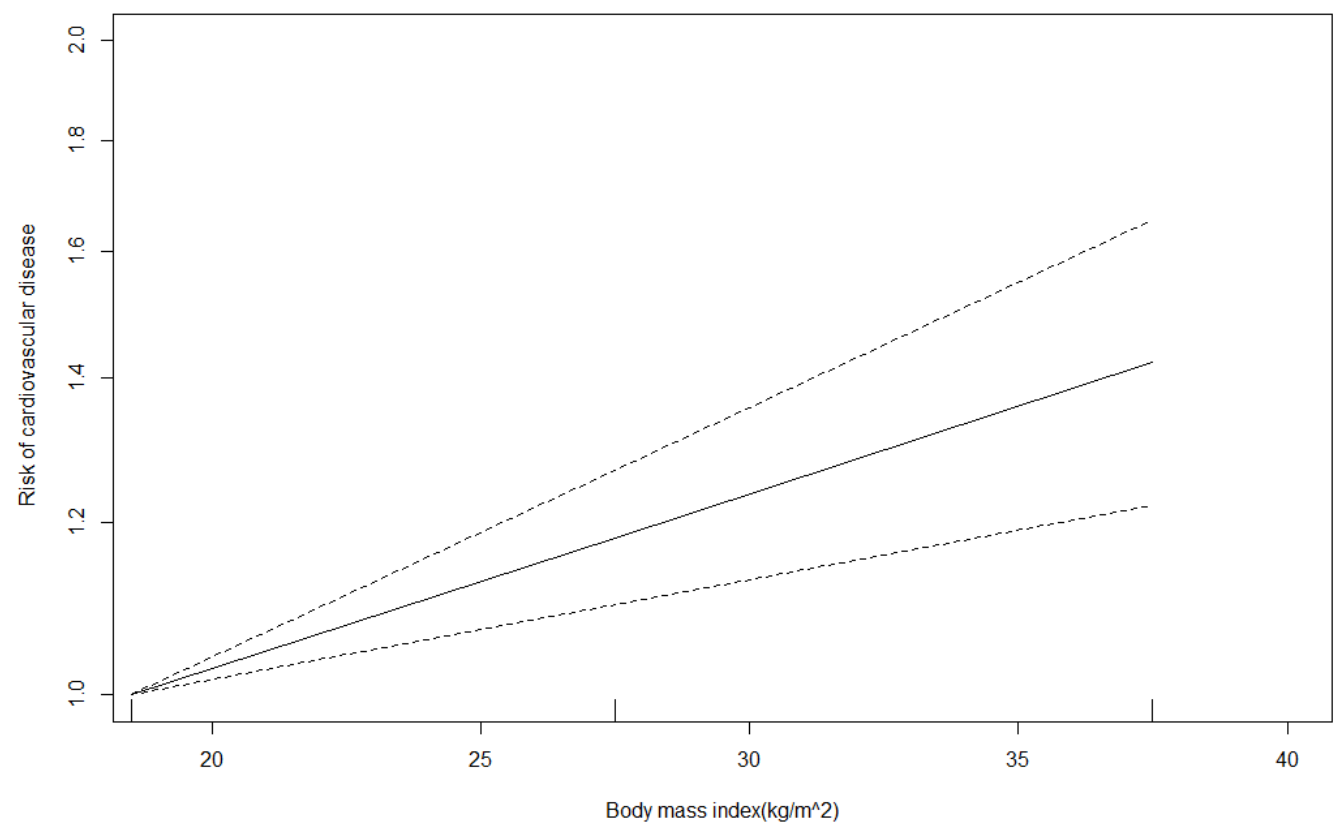

Figure 3. Dose-response analysis of body mass index and the risk of cardiovascular disease.

To explain the residual heterogeneity and to better understand the potential effect modifier, we performed prespecified meta-regression analyses of sex, age, follow-up duration and smoking status (Table 2). Univariate meta-regression showed that the risk of CVD due to MHO was borderline nonsignificant when modified by age, proportion of women, and smoking (bubble plots are shown in Figure 4 and Figures S4 and S5). Multivariate meta-regression model analysis showed that as the proportion of women and mean age increased, the impact of MHO on the risk of CVD diminished significantly. Smoking and follow-up duration did not modify the effect significantly (Figure S6). The proportion of heterogeneity explained by the meta-regression is represented by $R^{2}$. Age, sex, and smoking accounted for $99.99 \%$ of heterogeneity in terms of MHO and the risk of CVD. The funnel plot showed no substantial asymmetry, and Egger's test indicated no publication bias ( $p=0.73$; Figure S7). We excluded articles with Newcastle-Ottawa Scale scores below 7, omitted each study individually and excluded CV mortality from CV morbidity to perform sensitivity analyses. Overall, these statistics indicated that the results were robust (Table S3 and Figure S8). 


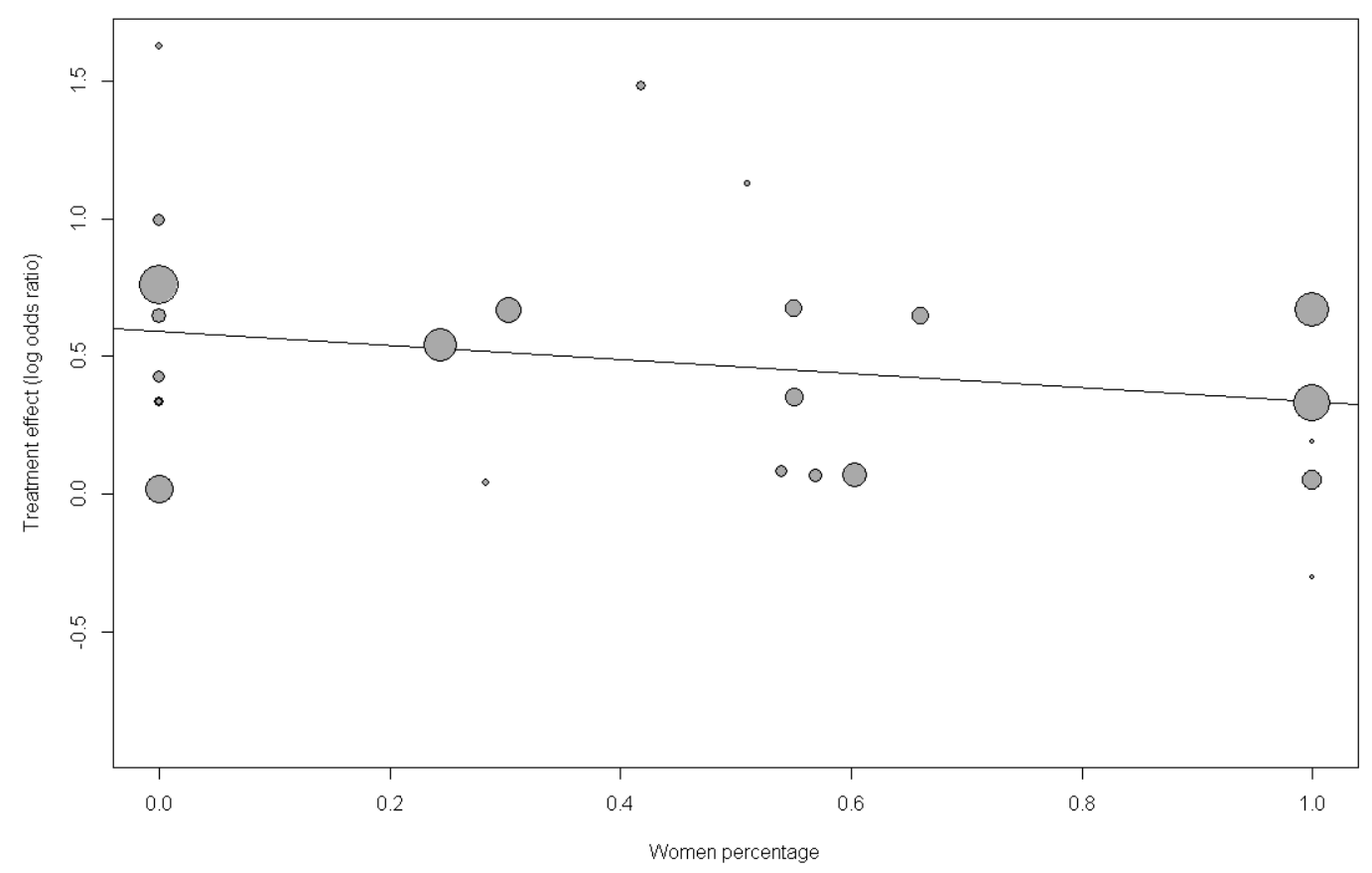

Figure 4. Meta-regression bubble plot of correlation between log odds ratio of cardiovascular disease and the proportion of women.

Each bubble represents a study and bubble size represents the sample size of the study. The regression line shows a nonsignificant trend of declining risk with larger women proportion. $\mathrm{OR}=0.77(0.50 ; 1.19), p=0.23, R^{2}(\%)=0 \%$.

A total of 20 cohort studies were pooled to evaluate the secondary outcome of all-cause mortality (Table 1). The cumulative forest plot showed an increased risk of all-cause mortality since 2005 (Figure S9). Participants with MHO had significantly higher rates of all-cause mortality than participants with MHNW. The subgroup analysis showed that, compared with participants with MHNW, those with MHO that was defined by insulin resistance had significantly higher rates of all-cause mortality. Participants with MHO defined by the modified ATP III criteria had borderline increased all-cause mortality. The modified JIS criteria or other definitions did not give similar results for all-cause mortality (the forest plot is shown in Figure S10). The subgroup analysis revealed that participants with MHO, defined by either WC or BMI, were at a significantly higher risk for all-cause mortality (the forest plot is shown in Figure S11).

A total of 9 articles with 26 BMI categories were pooled for dose-response analysis, which revealed a nonsignificant linear relationship between BMI and the risk of all-cause mortality (OR for slope, 1.01; $p=0.29$; Figure S12). The univariate meta-regression analysis revealed that age, smoking status and follow-up duration were borderline and nonsignificant effect modifiers $(p=0.07, p=0.77$ and $p=0.33$, respectively; bubble plots are shown in Figures S13-S15). Both univariate and multivariate meta-regression analyses indicated that as the proportion of women increased, the impact of MHO on the risk of all-cause mortality decreased significantly $(p=0.043$ and $p=0.015$, respectively; bubble plots are shown in Figure S16). Among sexes, the risk of all-cause mortality associated with MHO decreased significantly as age increased $(p=0.020)$. Age and sex accounted for $95.5 \%$ of heterogeneity in terms of MHO and the risk of all-cause mortality (Table S4). The funnel plot was symmetrical, and Egger's test indicated no significant publication bias ( $p=0.62$, Figure S17). We excluded articles with Newcastle-Ottawa Scale scores below 7 and omitted each study individually to perform sensitivity analyses. T results remained robust (Table S3 and Figure S18). 
Table 2. Meta-regression Analysis of Association between Covariates and Risk of Cardiovascular Disease.

\begin{tabular}{|c|c|c|c|c|c|c|c|c|}
\hline & \multicolumn{8}{|c|}{ Risk of Cardiovascular Disease } \\
\hline & \multicolumn{4}{|c|}{ Univariate } & \multicolumn{4}{|c|}{ Multivariate } \\
\hline & Unadjusted OR (95\% CI) & $p$ Value & $I^{2}(\%)$ & $R^{2}(\%)$ & Adjusted OR (95\% CI) & $p$ Value & $I^{2}(\%)$ & $R^{2}(\%)$ \\
\hline \multicolumn{9}{|l|}{ Sex } \\
\hline Men & 1 (Reference) & NA & & & 1 (Reference) & NA & & \\
\hline Women & $0.77(0.50 ; 1.19)$ & 0.23 & 59.3 & 0 & $0.65(0.46 ; 0.90)$ & 0.014 & 59.3 & 0 \\
\hline Age, per year increase & $0.99(0.97 ; 1.00)$ & 0.06 & 60.6 & 1.6 & $0.98(0.97 ; 1.00)$ & 0.030 & $39.1^{1}$ & $28.5^{1}$ \\
\hline \multicolumn{9}{|l|}{ Smoke status } \\
\hline Nonsmoker & 1 (Reference) & NA & & & 1 (Reference) & NA & & \\
\hline Smoker & $1.00(0.99 ; 1.01)$ & 0.94 & 65.5 & 0 & $1.01(1.00 ; 1.02)$ & 0.17 & $0^{2}$ & $99.99^{2}$ \\
\hline Follow-up duration, per year increase & $1.01(0.99 ; 1.03)$ & 0.43 & 64.5 & 2.2 & $1.00(0.97 ; 1.02)$ & 0.69 & $41.1^{3}$ & $18.1^{3}$ \\
\hline
\end{tabular}

$\mathrm{CI}$, confidence interval; NA, not applicable; OR, odds ratio. Bold font represents statistically significant results. ${ }^{1}$ Covariates: female sex and age. ${ }^{2}$ Covariates: female sex, age, and smoker ${ }^{3}$ Covariates: female sex, age and follow-up duration. 


\section{Discussion}

Our analysis demonstrates that individuals with $\mathrm{MHO}$ are at significantly higher risk of CVD and all-cause mortality, with BMI, sex, age, and smoking habits influencing the risk in such individuals.

Our results are consistent with three previous meta-analyses that reported that MHO caused the risk of CVD to increase significantly by $45 \%$ to $100 \%[23,24,40]$ compared with MHNW. A recent meta-analysis included only 21 studies and did not find a significant risk of CVD associated with MHO [26]. All-cause mortality was only discussed in one study included in the present meta-analysis, but the association with MHO was not found to be significant [24]. Compared with previous meta-analyses, we identified more studies and reported a more accurate pooled effect size and more precise $95 \% \mathrm{CI}$. This can be attributed to our more up-to-dated criteria and comprehensive approach to database searches.

Despite using different definitions of $\mathrm{MHO}$, the included studies consistently defined metabolic syndrome with the core concept of insulin resistance. In our study, we observed a higher risk of CVD when $\mathrm{MHO}$ was defined by insulin resistance than when other definitions were considered. The low heterogeneity that we observed may be attributed to consistency in the method of homeostasis model assessment (HOMA) among the studies. HOMA is a method used to quantify insulin resistance and beta cell function, computed as the product of fasting plasma glucose (FPG, $\mathrm{mmol} / \mathrm{L}$ ) and fasting serum insulin (mU/L) divided by 22.5. Lower values indicate high insulin sensitivity; whereas higher values indicate low insulin sensitivity or insulin resistance. Across our included studies, the cut-off value was based on a definite value or the quartile of the distribution among cohorts. The ATP III system [18] was found to be the most widely used, which defines metabolic syndrome as the presence of any three of the following traits: abdominal obesity defined as WC $\geq 102 \mathrm{~cm}$ in men or $\geq 88 \mathrm{~cm}$ in women; serum triglycerides $\geq 150 \mathrm{mg} / \mathrm{dL}$ or drug treatment for elevated triglycerides; serum high-density lipoprotein cholesterol (HDL-C) $<40 \mathrm{mg} / \mathrm{dL}(1.03 \mathrm{mmol} / \mathrm{L})$ in men or $<50 \mathrm{mg} / \mathrm{dL}$ in women or drug treatment for low HDL; blood pressure $\geq 130 / 85 \mathrm{mmHg}$ or drug treatment for elevated blood pressure; FPG $\geq 100 \mathrm{mg} / \mathrm{dL}$ or drug treatment for elevated blood glucose [41]. The IDF updated their metabolic syndrome criteria in 2006. Central obesity with ethnicity-specific WC cutoff points is an essential criterion, plus any two of the four following traits: triglycerides $\geq 150 \mathrm{mg} / \mathrm{dL}$ or treatment for elevated triglycerides; HDL-C $<40 \mathrm{mg} / \mathrm{dL}$ in men or $<50 \mathrm{mg} / \mathrm{dL}$ in women or treatment for low HDL; systolic blood pressure $\geq 130 \mathrm{mmHg}$, diastolic blood pressure $\geq 85 \mathrm{mmHg}$ or treatment for hypertension; FPG $\geq 100 \mathrm{mg} / \mathrm{dL}$ or previously diagnosed type 2 diabetes (an oral glucose tolerance test is recommended, but not required, for patients with elevated FPG) [19]. The latest JIS definition was proposed in 2009 and includes the same variables as the IDF criteria, although central obesity is not an essential component [20]. The studies included in the present analysis were found to use modified versions of the above criteria by using substituted or adjusted cutoff values of WC or FPG (or no cutoffs). Some studies used different definitions of metabolic syndrome, such as biomarkers including high-sensitivity C-reactive protein or apolipoprotein B, [42] whereas some defined the condition by diagnosis or treatment for hypertension, dyslipidemia, or diabetes. These modifications to the criteria demonstrated the inconsistent definitions of $\mathrm{MHO}$, which could be part of the within-study variance. Furthermore, one article reported the risk of all-cause mortality to be lower in patients with chronic kidney disease with MHO [43]; the underlying clinical differences may have contributed to this discrepancy with our results and the heterogeneity of our analysis. A single study showed a decreased risk of $\mathrm{HF}$ in participants with $\mathrm{MHO}$, although the small sample size of only 550 participants may mean that firm conclusions cannot be drawn [44].

The prevalence of MHO in our study was consistent with a previous report of an overall incidence of $7.3 \%$ [17]. None of our included studies reported the sex-specific impact at the individual-level. We found age and sex to be significant effect modifiers in the study-level. Although sex has been reported to play a role in the developmental programming of metabolism, [45] there are insufficient studies supporting our finding of the influence of sex. However, one study that focused on the outcome of hypertension showed a sex-specific impact of MHO [46]. Subgroup analyses performed as part of previous meta-analyses were underpowered to differentiate the modifying effects of sex, [26] smoking 
status, [24] and age [24]. The negative slope of age revealed that young participants with MHO had more risk of CVD than elderly participants with MHO, which may be related to sarcopenia and underlying diseases in the elderly. Early intervention of weight reduction is encouraged. More studies focusing on effect modifiers for the outcome of CVD in MHO are warranted.

The pathophysiology of MHO is considered as subclinical adipose tissue inflammation that often results in insulin resistance and is measured by predictors including C-reactive protein, interleukin 6, and free fatty acid levels; transition of adipose tissue leading to a metabolic state; and regulatory genetic predisposition involving the processes of apoptosis, adipogenesis, angiogenesis, and dysregulation of epigenetic adaptation hypothesis [47]. Metabolic support with D-ribose, coenzyme Q10, L-carnitine, and magnesium can improve the maintenance of contractile reserve and energy charge in minimally oxidative ischemic or hypoxic heart tissues [48]. Indirect evidence has revealed that supplementation with these nutrients may be helpful in maintaining heart function and weight loss [49,50]. Metabolic dysfunction in heart tissues may also explain the mechanism underlying the risk of CVD in MHO.

All phenotypes of obesity are considered to represent a state of disease. Individuals with MHO should not be considered as healthy, but as being in a "pre-metabolic syndrome" state [51] and at risk of future metabolic dysregulation or obesity-related health consequences. The concept of MHO seems most relevant in individuals who have mild, or class I, obesity (defined as BMI of 30 to <35) [17]. Moderate weight loss can reduce many unfavorable physiologic changes that are associated with obesity, and the cardiometabolic risks can be lowered. Therefore, every individual with obesity should be encouraged to achieve a normal weight in the long term [21].

The strength or the present study is that we employed a comprehensive search with strict definitions. Our analysis included millions of participants with long-term follow-up data from good-quality studies. However, our study does have several limitations that should be acknowledged. First, no randomized controlled studies were available and a causal relationship could not be established. We limited our analysis to cohort studies in order to establish temporality. Confounding factors in each study were adjusted using different models. The effect size of our results was moderate without publication bias, which is consistent with previous studies. Furthermore, our results indicate a plausible mechanism and coherence with epidemiological findings, as dose-response meta-analysis showed a significant linear association between BMI and the risk of CVD. Second, although our study evaluated obesity defined by various criteria, most of the included studies evaluated obesity by BMI only. Recent publications have suggested that the use of BMI alone is not a suitable indicator of obesity [52,53]. More research using indicators other than BMI is required. Third, the relationship between metabolically healthy underweight status and CVD is unknown; there is insufficient evidence to ascertain whether a J-shaped relationship exists between weight and CVD risk. Fourth, our included populations were of different ethnicities and from different centers. This diversity may not only be related to different definitions of obesity and metabolic health, but also related to the different CVD outcomes.

\section{Conclusions}

We provide robust evidence of a significant association of MHO with increased risk of CVD. Every incremental increase in BMI linearly increases this risk further. Therefore, all individuals with obesity should be encouraged to achieve a normal weight as early as possible and weight gain should also be discouraged in non-obese individuals.

Supplementary Materials: The following are available online at http://www.mdpi.com/2077-0383/8/8/1228/s1, Table S1: Newcastle-Ottawa scale for assessment of quality of included cohort studies, Table S2: Characteristics of included cohort studies, Figure S1: A Forest plot of cardiovascular disease, comparing participants with metabolically healthy obesity and participants with metabolically healthy normal weight, with a subgroup analysis by definitions of metabolic health, Figure S2: A Forest plot of cardiovascular disease, comparing participants with metabolically healthy obesity and participants with metabolically healthy normal weight, with a subgroup analysis by definitions of obesity, Figure S3: A Forest plot of cardiovascular disease, comparing participants with metabolically healthy obesity and participants with metabolically healthy normal weight, with a subgroup analysis by different cardiovascular outcomes, Figure S4: Meta-regression bubble plot of the correlation between $\log$ odds ratio of cardiovascular disease and age, Figure S5: Meta-regression bubble plot of the correlation between 
log odds ratio of cardiovascular disease and smoke, Figure S6: Meta-regression bubble plot of the correlation between log odds ratio of cardiovascular disease and follow-up duration, Figure S7: The funnel plot standard error and odds ratio of cardiovascular disease showing study dispersion, Table S3: Sensitivity analyses of article quality association between people with metabolically healthy obesity and risk of cardiovascular disease and all-cause mortality, Figure S8: Sensitivity analyses of metabolically healthy obesity and risk of cardiovascular disease by omitting each study, Figure S9: Cumulative forest plot of risk of all-cause mortality from all included studies, Figure S10: A Forest plot of all-cause mortality, comparing participants with metabolically healthy obesity and participants with metabolically healthy normal weight, with a subgroup analysis by definitions of metabolic health, Figure S11: A Forest plot of all-cause mortality, comparing participants with metabolically healthy obesity and participants with metabolically healthy normal weight, with a subgroup analysis by definitions of obesity, Figure S12: Dose-response analysis of body mass index and the risk of all-cause mortality, Figure S13: Meta-regression bubble plot of the correlation between log odds ratio of all-cause mortality and age, Figure S14: Meta-regression bubble plot of the correlation between log odds ratio of all-cause mortality and smoke, Figure S15: Meta-regression bubble plot of the correlation between log odds ratio of all-cause mortality and follow-up duration, Figure S16: Meta-regression of log odds ratio for all-cause mortality in people with metabolically healthy obesity relative to women proportion, Table S4: Meta-Regression analysis of association between covariates and risk of all-cause mortality, Figure S17: The funnel plot standard error and odds ratio of all-cause mortality showing study dispersion, Figure S18 Sensitivity analyses of metabolically healthy obesity and risk of all-cause mortality by omitting each study.

Author Contributions: All authors had full access to the data in the study and take responsibility for the integrity of the data and the accuracy of the data analysis. T.-L.Y. formulated the study objective, conceptualized the study, performed the statistical analysis, and interpreted the results; S.-J.L. explored resources and performed the methodology. T.-L.Y., H.-H.C., S.-Y.T. and C.-Y.L. performed the methodology, data collection, and data validation; T.-L.Y. helped with the formal statistical analysis and analysis of the data; T.-L.Y. and K.-L.C. contributed to outlining the manuscript, developing the study design, and drafting the manuscript; K.-L.C. supervised and coordinated the study. All authors have read and approved the final version.

Acknowledgments: We would like to offer our thanks to Jing-Rong, Jhuan and Hua, Li, the Institute of Epidemiology and Preventive Medicine, National Taiwan University and Yi-Chun Yeh, Postdoctoral Fellow of Department of Medical Research, National Taiwan University Hospital, for statistics. We offer our thanks to the MacKay Memorial Hospital librarian, Pei-jin Li, for examining the references. We would like to thank Editage (www.editage.com) for editing and proofreading this manuscript.

Conflicts of Interest: The authors declare no conflict of interest.

\section{References}

1. World Health Organization. Obesity: Preventing and Managing the Global Epidemic. Report of a WHO Consultation; World Health Organization Technical Report Series 894; World Health Organization: Geneva, Switzerland, 2000; Volume 894, i-xii, pp. 1-253.

2. O'Rourke, K.; VanderZanden, A.; Shepard, D.; Leach-Kemon, K.; Institute for Health Metrics and Evaluation. Cardiovascular disease worldwide, 1990-2013. JAMA 2015, 314, 1905. [CrossRef]

3. Adams, K.F.; Schatzkin, A.; Harris, T.B.; Kipnis, V.; Mouw, T.; Ballard-Barbash, R.; Hollenbeck, A.; Leitzmann, M.F. Overweight, obesity, and mortality in a large prospective cohort of persons 50 to 71 years old. N. Engl. J. Med. 2006, 355, 763-778. [CrossRef] [PubMed]

4. Jee, S.H.; Sull, J.W.; Park, J.; Lee, S.Y.; Ohrr, H.; Guallar, E.; Samet, J.M. Body-mass index and mortality in korean men and women. N. Engl. J. Med. 2006, 355, 779-787. [CrossRef] [PubMed]

5. McTigue, K.; Larson, J.C.; Valoski, A.; Burke, G.; Kotchen, J.; Lewis, C.E.; Stefanick, M.L.; Van Horn, L.; Kuller, L. Mortality and cardiac and vascular outcomes in extremely obese women. JAMA 2006, 296, 79-86. [CrossRef] [PubMed]

6. Yan, L.L.; Daviglus, M.L.; Liu, K.; Stamler, J.; Wang, R.; Pirzada, A.; Garside, D.B.; Dyer, A.R.; Van Horn, L.; Liao, Y.; et al. Midlife body mass index and hospitalization and mortality in older age. JAMA 2006, 295, 190-198. [CrossRef] [PubMed]

7. Ortega, F.B.; Lavie, C.J.; Blair, S.N. Obesity and cardiovascular disease. Circ. Res. 2016, 118, $1752-1770$. [CrossRef] [PubMed]

8. Bosello, O.; Donataccio, M.P.; Cuzzolaro, M. Obesity or obesities? Controversies on the association between body mass index and premature mortality. Eat. Weight Disord. 2016, 21, 165-174. [CrossRef]

9. Eftekharzadeh, A.; Asghari, G.; Serahati, S.; Hosseinpanah, F.; Azizi, A.; Barzin, M.; Mirmiran, P.; Azizi, F. Predictors of incident obesity phenotype in nonobese healthy adults. Eur. J. Clin. Invest. 2017, 47, 357-365. [CrossRef] 
10. Hainer, V.; Zamrazilová, H.; Aldhoon-Hainerová, I. Metabolically healthy obesity: Does it exist? Diabetol. Metab. Endokrinol. Vyziva 2015, 18, 112-120.

11. Phillips, C.M. Metabolically healthy obesity: Definitions, determinants and clinical implications. Rev. Endocr. Metab. Disord. 2013, 14, 219-227. [CrossRef]

12. Stefan, N.; Haring, H.U.; Hu, F.B.; Schulze, M.B. Metabolically healthy obesity: Epidemiology, mechanisms, and clinical implications. Lancet Diabetes Endocrinol. 2013, 1, 152-162. [CrossRef]

13. De Lorenzo, A.; da Cruz Lamas, C.; Lessa, R.; Moreira, A.S.B. “Metabolically healthy” obesity: Fact or threat? Curr. Diabetes Rev. 2018, 14, 405-410. [CrossRef] [PubMed]

14. Roberson, L.L.; Aneni, E.C.; Maziak, W.; Agatston, A.; Feldman, T.; Rouseff, M.; Tran, T.; Blaha, M.J.; Santos, R.D.; Sposito, A.; et al. Beyond bmi: The "metabolically healthy obese" phenotype \& its association with clinical/subclinical cardiovascular disease and all-cause mortality-A systematic review. BMC Public Health 2014, 14, 14.

15. Sims, E.A. Are there persons who are obese, but metabolically healthy? Metabolism 2001, 50, 1499-1504. [CrossRef] [PubMed]

16. Stefan, N.; Kantartzis, K.; Machann, J.; Schick, F.; Thamer, C.; Rittig, K.; Balletshofer, B.; Machicao, F.; Fritsche, A.; Häring, H.U. Identification and characterization of metabolically benign obesity in humans. Arch. Int. Med. 2008, 168, 1609-1616. [CrossRef] [PubMed]

17. Phillips, C.M. Metabolically healthy obesity across the life course: Epidemiology, determinants, and implications. Ann. N. Y. Acad. Sci. 2017, 1391, 85-100. [CrossRef] [PubMed]

18. Expert Panel on Detection, Evaluation; Treatment of High Blood Cholesterol in Adults. Executive summary of the third report of the national cholesterol education program (ncep) expert panel on detection, evaluation, and treatment of high blood cholesterol in adults (adult treatment panel III). JAMA 2001, 285, 2486-2497. [CrossRef] [PubMed]

19. Alberti, K.G.; Zimmet, P.; Shaw, J. Metabolic syndrome-A new world-wide definition. A consensus statement from the international diabetes federation. Diabet. Med. 2006, 23, 469-480. [CrossRef]

20. Alberti, K.G.; Eckel, R.H.; Grundy, S.M.; Zimmet, P.Z.; Cleeman, J.I.; Donato, K.A.; Fruchart, J.C.; James, W.P.; Loria, C.M.; Smith, S.C., Jr.; et al. Harmonizing the metabolic syndrome: A joint interim statement of the international diabetes federation task force on epidemiology and prevention; national heart, lung, and blood institute; american heart association; world heart federation; international atherosclerosis society; and international association for the study of obesity. Circulation 2009, 120, 1640-1645.

21. Stefan, N.; Haring, H.U.; Schulze, M.B. Metabolically healthy obesity: The low-hanging fruit in obesity treatment? Lancet Diabetes Endocrinol. 2018, 6, 249-258. [CrossRef]

22. Wang, B.; Zhuang, R.; Luo, X.; Yin, L.; Pang, C.; Feng, T.; You, H.; Zhai, Y.; Ren, Y.; Zhang, L.; et al. Prevalence of metabolically healthy obese and metabolically obese but normal weight in adults worldwide: A meta-analysis. Horm. Metab. Res. 2015, 47, 839-845. [CrossRef] [PubMed]

23. Eckel, N.; Meidtner, K.; Kalle-Uhlmann, T.; Stefan, N.; Schulze, M.B. Metabolically healthy obesity and cardiovascular events: A systematic review and meta-analysis. Eur. J. Prev. Cardiol. 2016, 23, 956-966. [CrossRef] [PubMed]

24. Zheng, R.; Zhou, D.; Zhu, Y. The long-term prognosis of cardiovascular disease and all-cause mortality for metabolically healthy obesity: A systematic review and meta-analysis. J. Epidemiol. Community Health 2016, 70, 1024-1031. [CrossRef] [PubMed]

25. Obesity, Metabolically Benign. Available online: https://www.ncbi.nlm.nih.gov/mesh/?term=metabolically+ healthy+obesity (accessed on 15 August 2019).

26. Mirzababaei, A.; Djafarian, K.; Mozafari, H.; Shab-Bidar, S. The long-term prognosis of heart diseases for different metabolic phenotypes: A systematic review and meta-analysis of prospective cohort studies. Endocrine 2019, 63, 439-462. [CrossRef] [PubMed]

27. Jung, C.H.; Lee, W.J.; Song, K.H. Metabolically healthy obesity: A friend or foe? Korean J. Intern. Med. 2017, 32, 611-621. [CrossRef] [PubMed]

28. Shamseer, L.; Moher, D.; Clarke, M.; Ghersi, D.; Liberati, A.; Petticrew, M.; Stewart, L.A. Preferred reporting items for systematic review and meta-analysis protocols (prisma-p) 2015: Elaboration and explanation. BMJ 2015, 350, g7647. [CrossRef] [PubMed]

29. Stang, A. Critical evaluation of the newcastle-ottawa scale for the assessment of the quality of nonrandomized studies in meta-analyses. Eur. J. Epidemiol. 2010, 25, 603-605. [CrossRef] 
30. Team, R.C. R: A Language and Environment for Statistical Computing; R Foundation for Statistical Computing: Vienna, Austria, 2019.

31. DerSimonian, R.; Laird, N. Meta-analysis in clinical trials. Control. Clin. Trials 1986, 7, 177-188. [CrossRef]

32. Higgins, J.P.; Thompson, S.G. Quantifying heterogeneity in a meta-analysis. Stat. Med. 2002, 21, 1539-1558. [CrossRef]

33. Greenland, S.; Longnecker, M.P. Methods for trend estimation from summarized dose-response data, with applications to meta-analysis. Am. J. Epidemiol. 1992, 135, 1301-1309. [CrossRef]

34. Rothman, K.J. Epidemiology: An Introduction; Oxford University press: Oxford, UK, 2012.

35. Egger, M.; Davey Smith, G.; Schneider, M.; Minder, C. Bias in meta-analysis detected by a simple, graphical test. BMJ 1997, 315, 629-634. [CrossRef] [PubMed]

36. Choi, K.M.; Cho, H.J.; Choi, H.Y.; Yang, S.J.; Yoo, H.J.; Seo, J.A.; Kim, S.G.; Baik, S.H.; Choi, D.S.; Kim, N.H. Higher mortality in metabolically obese normal-weight people than in metabolically healthy obese subjects in elderly koreans. Clin. Endocrinol. 2013, 79, 364-370. [CrossRef] [PubMed]

37. Kip, K.E.; Marroquin, O.C.; Kelley, D.E.; Johnson, B.D.; Kelsey, S.F.; Shaw, L.J.; Rogers, W.J.; Reis, S.E. Clinical importance of obesity versus the metabolic syndrome in cardiovascular risk in women: A report from the women's ischemia syndrome evaluation (wise) study. Circulation 2004, 109, 706-713. [CrossRef] [PubMed]

38. Kuk, J.L.; Ardern, C.I. Are metabolically normal but obese individuals at lower risk for all-cause mortality? Diabetes Care 2009, 32, 2297-2299. [CrossRef] [PubMed]

39. Li, L.; Chen, K.; Wang, A.P.; Gao, J.Q.; Zhao, K.; Wang, H.B.; Dou, J.T.; Lv, Z.H.; Wang, B.A.; Yan, W.H.; et al. Cardiovascular disease outcomes in metabolically healthy obesity in communities of beijing cohort study. Int. J. Clin. Pract. 2018, e13279. [CrossRef] [PubMed]

40. Fan, J.; Song, Y.; Chen, Y.; Hui, R.; Zhang, W. Combined effect of obesity and cardio-metabolic abnormality on the risk of cardiovascular disease: A meta-analysis of prospective cohort studies. Int. J. Cardiol. 2013, 168, 4761-4768. [CrossRef] [PubMed]

41. Grundy, S.M.; Cleeman, J.I.; Daniels, S.R.; Donato, K.A.; Eckel, R.H.; Franklin, B.A.; Gordon, D.J.; Krauss, R.M.; Savage, P.J.; Smith, S.C., Jr.; et al. Diagnosis and management of the metabolic syndrome: An american heart association/national heart, lung, and blood institute scientific statement. Circulation 2005, 112, 2735-2752. [CrossRef] [PubMed]

42. St-Pierre, A.C.; Cantin, B.; Mauriege, P.; Bergeron, J.; Dagenais, G.R.; Despres, J.P.; Lamarche, B. Insulin resistance syndrome, body mass index and the risk of ischemic heart disease. CMAJ 2005, 172, 1301-1305. [CrossRef]

43. Hanks, L.J.; Tanner, R.M.; Muntner, P.; Kramer, H.; McClellan, W.M.; Warnock, D.G.; Judd, S.E.; Gutiérrez, O.M. Metabolic subtypes and risk of mortality in normal weight, overweight, and obese individuals with ckd. Clin. J. Am. Soc. Nephrol. 2013, 8, 2064-2071. [CrossRef]

44. Voulgari, C.; Tentolouris, N.; Dilaveris, P.; Tousoulis, D.; Katsilambros, N.; Stefanadis, C. Increased heart failure risk in normal-weight people with metabolic syndrome compared with metabolically healthy obese individuals. J. Am. Coll. Cardiol. 2011, 58, 1343-1350. [CrossRef]

45. Dearden, L.; Bouret, S.G.; Ozanne, S.E. Sex and gender differences in developmental programming of metabolism. Mol. Metab. 2018, 15, 8-19. [CrossRef] [PubMed]

46. Kabootari, M.; Akbarpour, S.; Azizi, F.; Hadaegh, F. Sex specific impact of different obesity phenotypes on the risk of incident hypertension: Tehran lipid and glucose study. Nutr. Metab. (Lond) 2019, 16, 16. [CrossRef] [PubMed]

47. Acharya, S.; Shukla, S. Metabolic healthy obesity-A paradoxical fallacy? J. Clin. Diagn. Res. 2018, 12, OE07-OE10. [CrossRef]

48. Roberts, J.C.; Sinatra, S.T. The Sinatra Solution: Metabolic Cardiology; Basic Health Publications, Inc.: Laguna Beach, CA, USA, 2014.

49. Vest, A.R.; Chan, M.; Deswal, A.; Givertz, M.M.; Lekavich, C.; Lennie, T.; Litwin, S.E.; Parsly, L.; Rodgers, J.E.; Rich, M.W.; et al. Nutrition, obesity and cachexia in patients with heart failure: A consensus statement from the hfsa scientific statements committee. J. Card. Fail. 2019, 25, 380-400. [CrossRef] [PubMed]

50. Pooyandjoo, M.; Nouhi, M.; Shab-Bidar, S.; Djafarian, K.; Olyaeemanesh, A. The effect of (1-)carnitine on weight loss in adults: A systematic review and meta-analysis of randomized controlled trials. Obes. Rev. 2016, 17, 970-976. [CrossRef] [PubMed] 
51. Rasaei, N.; Mirzababaei, A.; Arghavani, H.; Tajik, S.; Keshavarz, S.A.; Yekaninejad, M.S.; Imani, H.; Mirzaei, K. A comparison of the sensitivity and specificity of anthropometric measurements to predict unhealthy metabolic phenotype in overweight and obese women. Diabetes Metab. Syndr. Clin. Res. Rev. 2018, 12, 1147-1153. [CrossRef] [PubMed]

52. De Lorenzo, A.; Gratteri, S.; Gualtieri, P.; Cammarano, A.; Bertucci, P.; Di Renzo, L. Why primary obesity is a disease? J. Transl. Med. 2019, 17, 169. [CrossRef] [PubMed]

53. Chrysant, S.G.; Chrysant, G.S. The single use of body mass index for the obesity paradox is misleading and should be used in conjunction with other obesity indices. Postgrad. Med. 2019, 131, 96-102. [CrossRef] [PubMed]

(C) 2019 by the authors. Licensee MDPI, Basel, Switzerland. This article is an open access article distributed under the terms and conditions of the Creative Commons Attribution (CC BY) license (http://creativecommons.org/licenses/by/4.0/). 